\title{
Testing Soluble Epoxide Hydrolase Inhibition in Patients with Aneurysmal Subarachnoid Hemorrhage: Rare Diseases, Orphan Drugs, the Conundrum
}

\author{
Paul Nyquist ${ }^{*}$ [
}

@ 2021 Springer Science+Business Media, LLC, part of Springer Nature and Neurocritical Care Society

The Orphan Drug Act (1988) defines a "rare disease" as a disease or condition that affects less than 200,000 people in the United States. With an incidence of 7.9 (95\% confidence interval 6.9-9.0) per 100,000 person-years [1] and an estimated 52,379 cases of aneurysmal subarachnoid hemorrhage (SAH) per year in the USA [2], SAH is a rare disease. If you consider the issue of the secondary consequences of SAH of delayed cerebral ischemia (DCI), it is even rarer, with approximately $30 \%$ of patients with $\mathrm{SAH}$ experiencing DCI [3]. In neurocritical care, DCI and SAH are considered important clinical problems and are commonly treated. Yet, these diseases are not common, and thus the adequate funding that would inspire discovery of treatments for DCI are yet to be forthcoming.

In this month's edition of Neurocritical Care, Martini et al. [4] report the initial results of a phase Ib trial of a soluble epoxide hydrolase inhibitor in the article entitled, "A Double-Blind, Randomized, Placebo-Controlled Trial of Soluble Epoxide Hydrolase Inhibition in Patients with Aneurysmal Subarachnoid Hemorrhage." This study was a phase Ib, randomized, double-blinded, placebo-controlled trial involving 19 patients admitted to the neurosciences intensive care unit at Oregon Health and Science University. The patients with SAH were randomly assigned to receive $10 \mathrm{mg}$ of GSK2256294

*Correspondence: pnyquis1@jhmi.edu

Departments of Neurology, Anesthesia/Critical Care, Medicine, Neurosurgery, and General Internal Medicine, Johns Hopkins School of Medicine, Baltimore, MD, USA

This article is related to the original article available at https://link.sprin ger.com/article/10.1007/s12028-021-01398-8

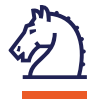

\section{Springer}

or a placebo once daily for 10 days, beginning within $72 \mathrm{~h}$ after aneurysm rupture. This study tests the effect of a new epoxide hydrolase inhibitor, GSK2256294, as a treatment for DCI. The drug is a known inhibitor of the soluble epoxide hydrolase that metabolizes the epoxyeicosatrienoates to dihydroxyeicosatrienoates (DHETs) and prevents pathway amplification of DHETs. The goal of the study was to determine the initial safety of GSK2256294 and establish initial protocols for its use in humans. The DHETs are endogenous regulators of neuroinflammation and cerebral blood flow. DHETs have been identified as important molecules affecting control of microvascular perfusion and have been suggested to play a role in Alzheimer disease and stroke. It is hypothesized that these molecules may contribute to DCI after SAH [5]. For over a decade, the inhibition of soluble epoxide hydrolase has been proposed as a potential target to downregulate eiconocosiods and DHET production and prevent DCI [6]. This study builds on this legacy of solid basic science research, with well-developed biological models ready for translation to humans.

Conducting clinical trials in rare diseases without adequate pharma or National Institutes of Health support often results in lower recruitment and smaller sample sizes, with a decreased ability to measure and observe key phenotypes. This study was conducted in a single center on a very tight budget and was slightly underpowered. Some of the issues addressed by a phase Ib study remain unclarified. These include the issues of observed adverse drug reactions and drug tolerability, specifically in DCI. That said, the authors and leaders of this small trial appear to have pulled it off. They have achieved 
most of their primary goals. The trial outlines the basis for a future larger trial. They have passed the phase Ib landmark of introductory human use with no significant observed adverse actions. Presently, they have now outlined a study protocol with well-defined phenotypic markers of DCI and vasoreactivity to include transcranial Doppler, cerebral angiograms, and clinical criterion for DCI. The authors have also measured DHETs as biomarkers in the serum and cerebro spinal fluid (CSF) of these patients to test for drug activity. They have detected a trend toward decreases in DHETs, suggesting the potential efficacy of GSK2256294. Furthermore, they have measured the epoxyeicosatrienoates-to-DHETs ratio in serum and CSF, allowing for an appropriate power calculation for future trails.

This study offers no new clinical insights and should not be taken as a guide to the clinical care of patients with SAH or for the prevention of DCI. It is, however, a well-conceived trial using a tested biological model and is the first necessary step in developing a new clinical tool. This is what must happen in neurocritical care if we are going to develop tools to treat our large subset of rare diseases. We cannot rely on retrospective clinical studies for clinical guidance. We cannot randomly apply presently approved drugs to new uses in a haphazard manner based on dubious expert opinion. We must move new scientific concepts and biological models into animal models and test them appropriately in human trials.
Source of support

This work received no funding.

Conflicts of interest

The author has no conflicts to disclose.

\section{Publisher's Note}

Springer Nature remains neutral with regard to jurisdictional claims in published maps and institutional affiliations.

Received: 16 November 2021 Accepted: 22 November 2021

Published: 9 December 2021

References

1. Etminan N, Chang HS, Hackenberg K, de Rooij NK, Vergouwen MDI, Rinkel GJE, et al. Worldwide incidence of aneurysmal subarachnoid hemorrhage according to region, time period, blood pressure, and smoking prevalence in the population: a systematic review and meta-analysis. JAMA Neurol. 2019;76:588-97.

2. McDonald RJ, McDonald JS, Bida JP, Kallmes DF, Cloft HJ. Subarachnoid hemorrhage incidence in the United States does not vary with season or temperature. AJNR Am J Neuroradiol. 2012;33:1663-8.

3. van Gijn J, Kerr RS, Rinkel GJ. Subarachnoid haemorrhage. Lancet. 2007:369:306-18.

4. Martini R, Silter D, Cetas J, Alkayed N, Treggiari M. A double-blind, randomized, placebo-controlled trial of soluble epoxide hydrolase inhibition in patients with aneurysmal subarachnoid hemorrhage. Neurocrit Care. 2021. https://doi.org/10.1007/s12028-021-01398-8.

5. Poloyac SM, Reynolds RB, Yonas H, Kerr ME. Identification and quantification of the hydroxyeicosatetraenoic acids, 20-HETE and 12-HETE, in the cerebrospinal fluid after subarachnoid hemorrhage. J Neurosci Methods. 2005;144:257-63.

6. Zhang W, Koerner IP, Noppens R, Grafe M, Tsai HJ, Morisseau C, et al. Soluble epoxide hydrolase: a novel therapeutic target in stroke. J Cereb Blood Flow Metab. 2007;27:1931-40. 